ISSN-i 1390-7042 | IsSN-e 2602-8484

\title{
ARQUITECTURA ESTRATIGRÁFICA DE TERRAZAS ALUVIALES EN EL RÍO YUNGANZA
}

\section{Stratigraphic architecture of alluvial terraces in the Yunganza river}

Wladimir Romero Cóndor Instituto de Investigación Geológico y Energético wladimir.romero@geoenergia.gob.ec ORCID: https://orcid.org/0000-0001-9271-4323

Fausto Carranco Andino Instituto de Investigación Geológico y Energético fausto.carranco@geoenergia.gob.ec ORCID: https://orcid.org/0000-0001-9412-8942

\section{Andrea Cisneros Medina}

andrea.cisneros.medina@gmail.com oRCID: https://orcid.org/0000-0003-1347-8479

Ana Belén Gramal Aguilar

Instituto de Investigación Geológico y Energético ana.gramal@geoenergia.gob.ec

ORCID: https://orcid.org/0000-0002-2216-8846
Darwin Condoy Guiracocha

Instituto de Investigación Geológico y Energético darwin.condoy@geoenergia.gob.ec

oRCID: https://orcid.org/0000-0001-5783-8347

Franz Betancourt Valdivieso

Instituto de Investigación Geológico y Energético franz.betancourt@geoenergia.gob.ec

ORCID: https://orcid.org/0000-0001-8306-6563
Recibido: 19 de agosto de 2020 Aprobado: 18 de diciembre de 2020 ARTÍCULO ORIGINAL

DoI: $10.29166 /$ revfig.vli2.2482
PALABRAS ClAve Terrazas aluviales, sedimentología fluvial, río Yunganza.

KEYWORDS Alluvial terraces, fluvial sedimentology, Yunganza river.

\section{RESUMEN}

El estudio de patrones de sedimentación fluvial (PSF) permite analizar el comportamiento tectónico y climático en una determinada región, además entender cómo los ríos modifican el relieve. Los PSF pueden ser estudiados a partir del análisis del registro estratigráfico de sus depósitos. En los Andes del norte, los PSF interactúan con tasas de erosión y generación de detrito disponible para la sedimentación. En el sur, del levantamiento subandino ecuatoriano, la cuenca del río Yunganza es una cuenca fluvial confinada, alimentadora del río Santiago y ofrece la posibilidad de estudiar los PSF de esta región. El objetivo de esta investigación es presentar el análisis del registro estratigráfico de terrazas aluviales antiguas (TA-1 y TA-2) y depósitos aluviales modernos (DA), en la cuenca alta del río Yunganza, en el sector El Rosario, con el fin de definir PSF. Esta investigación es soportada por la documentación del registro litológico, las estructuras sedimentarias, la arquitectura estratigráfica, la medición de espesores y paleocorrientes. De esta manera, 7 facies son descritas: LT- 1 y LT- 2 describen a conglomerados asociados a flujos de escombros distales, mientras que LT-3 describe litologías de conglomerados y brechas asociadas a flujos de escombros proximales. LT-4, LT-5 y LT-6 describen litologías arenosas vinculadas genéticamente a macroformas de barras y canales fluviales; LT-7 describe litologías limosas que se asocian a planicies de inundación. Datos de paleocorrientes indican que: TA-1 presenta una dirección de flujo N-NE, mientras que TA-2 indican una dirección de transporte de sedimento hacia el $\mathrm{N}$. La composición modal en TA-1 indica una procedencia detrítica de un bloque sedimentario rico en cuarzo, mientras que TA-2 indica un periodo de reciclaje de TA-1. La medición de espesores en TA-1 y TA-2 indica un periodo erosivo en TA-1. Finalmente, los patrones de sedimentación en el río Yunganza están vinculados genéticamente a periodos de exhumación del flanco oeste del río.

\section{ABSTRACT}

The study of fluvial sedimentation patterns (FSP) allow us to analyze tectonic and climatic behavior in a specific region, and, understand how rivers modify 
the relief. FSP can be studied from the analysis of the stratigraphic record of their deposits. In the northern Andes, FSP interact with erosion rates and detritus available for sedimentation rates. In the south of the sub-Andean zone, the Yunganza river basin corresponds to a confined fluvial basin and feeder of the Santiago river, and offers the opportunity to study this region FSP. The aim of this research is to present the stratigraphic record analysis of: ancient alluvial terraces (TA-1 and TA-2) and alluvial deposits (DA) in the Yunganza river basin, in the vicinity of El Rosario in order to define PSF. Our research is supported by documentation of the lithological record, sedimentary structures, stratigraphic architecture, thickness and paleo-currents measurement. Seven facies are described: LT-1 and LT-2 describe conglomerates associated with distal debris flows, whilst, LT-3 describes conglomerates and breccias associated with proximal debris flows. LT-4, and LT-5, and LT-6 describe sandstones genetically linked to bars and fluvial channel macro-forms, last, LT-7 describes siltstones associated with flood plains. Paleocurrent data indicate that: TA-1 presents flow direction to NE-E, whereas, TA-2 indicates a sediment transport direction to N. The TA-1 modal composition indicates a detrital provenance of a sedimentary block rich in quarts, while the TA-2 modal composition indicates a recycling period of TA1. The thickness measurement in TA-1 and TA-2 indicates an intensely erosive period in TA-1. Finally fluvial sedimentation patterns in the Yunganza river are genetically linked to periods of exhumation on the west side of the river.

\section{INTRODUCCIÓN}

El estudio de procesos de sedimentación en cuencas fluviales se desarrolló a nivel mundial, debido a la importancia cultural y económica de los ríos para el desarrollo de la sociedad (Bull, 1977). En los cinturones montañosos, procesos de denudación y erosión son controlados por tasas de exhumación de cuerpos rocosos y el clima (Jolley et al., 1990). La erosión controla el relieve y patrones de sedimentación (Lewin et al., 2005). El estudio de patrones de sedimentación fluvial en cinturones montañosos incluye: la descripción de la arquitectura estratigráfica de terrazas aluviales en cuencas confinadas (Benito et al., 2000).

En las estribaciones de cinturones montañosos como Himalayas, Alpes, Andes se han documentado el desarrollo de cuencas fluviales confinadas en las cuales patrones erosivos transportan volúmenes kilométricos de detrito hacia planicies en el transarco o el antearco, construyendo grandes abanicos aluviales que caracterizan la transición desde cinturones montañosos a tierras bajas (Fontana,et al., 2014; Ventra y Clarke, 2018). Si bien es cierto, la génesis de estos abanicos aluviales ha sido ampliamente estudiada, el patrón de generación de detrito disponible en cuencas confinadas alimentadoras de estos abanicos aluviales, no es tan claro y en la actualidad este se convierte en un tema de interés para la comunidad geocientífica (Weissmann et al., 2010).

Los Andes son el clásico ejemplo de un cinturón montañoso construido a lo largo de una zona de subducción
(Pratt et al., 2005). Ecuador se localiza al sur de los Andes del norte, en el límite de placas convergentes, en donde la cordillera submarina de Carnegie se subduce en la placa sudamericana a una tasa media de $58 \mathrm{~mm} / \mathrm{a}$ (ver Figura 1A); las características de los procesos de subducción controlan la tasa de elevación y subsidencia a nivel regional (Sage et al., 2006).

En el Ecuador, los Andes mantienen una tendencia $\mathrm{N}$-NE y se componen de dos distintas cordilleras. El basamento de la cordillera occidental y la planicie costera es considerado un terreno oceánico alóctono acrecionado a la margen noroccidental sudamericana a lo largo de la falla Calacali-Pallatanga-Palenque (CPPF); (Aspden y Litherland, 1992). Las fallas Las Aradas (LAF) y Raspas (RF) separan a la cordillera Real del Bloque Amotape Tahuín (BAT). (Riel et al., 2013) sugiere que el BAт corresponde a un slab rotado de la cordillera Real (ver Figura 1A).

La falla Peltetec (PF) representa el límite occidental de la cordillera Real, la cual es considerada como un cinturón de rocas metamórficas precámbricas a mesozoicas. Mientras que el sistema de fallas Cosanga (CF), Méndez (MF), Palanda (PLF) ha sido interpretado como el límite oriental de la cordillera (Litherland, 1994). Al E del sistema de fallas CF-MF-PLF, la región subandina corresponde a un cinturón de orientación $\mathrm{N}-\mathrm{s}$, deformado, corrido y plegado; de $\mathrm{N}$ a s, se compone del levantamiento Napo, depresión Pastaza y la cordillera Cutucú (Baby et al., 2013). 
Al sw de la cordillera Cutucú, en el flanco e de la cordillera Real, la cuenca del río Yunganza es una cuenca fluvial de segundo orden que alimenta al río Namangoza tributario del río Zamora. Esta cuenca es controlada por el movimiento de bloques tectónicos que afectan a secuencias cretácicas (ver Figura 1).

En este contexto, la falla Copal corresponde a un sistema de fallas inversas de dirección N-S, buzando al w, que afecta a rocas de la Formación Napo. El sistema de fallas Yunganza-Numbaime corresponde a fallas inversas de dirección, NNE-NE, con buzamiento al SE, estas fallas ponen en contacto tectónico a las formaciones Hollín y Misahuallí. La falla El Castillo corresponde a una falla inversa, de dirección NE-SW, que buza al Nw y pone en contacto tectónico a rocas de las formaciones Hollín y Napo. La falla Limón es una falla inversa de dirección NE-sw, que buza al NW $y$ afecta a secuencias terciarias, poniendo en contacto bloques de la Formación Hollín y Terrazas Aluviales (ver Figuras 1B y 2)

La zona de estudio fue definida al s del poblado El Rosario, debido a la presencia de afloramientos de grandes dimensiones y con buena continuidad lateral que permiten estudiar depósitos aluviales antiguos que en la actualidad forman estructuras en mesa o terrazas aluviales y depósitos aluviales modernos a lo largo del río Yunganza (ver Tabla 1).

Esta investigación presenta la documentación de características litológicas, estructuras sedimentarias, arquitectura estratigráfica, tendencias en la variación del tamaño de estrato, con el fin de proponer ambientes de depósito. Esta investigación incluyó el levantamiento de datos topográficos con ayuda de GPS diferencial, con el fin de evaluar el espesor de estos depósitos. De esta manera, este estudio analiza la generación de detrito disponible y patrones de sedimentación en la cuenca confinada del río Yunganza, que alimenta al río Santiago

\section{METODOLOGÍA}

Esta investigación utiliza la identificación, descripción e interpretación de facies sedimentarias y considera el término facie definido por Walker (1990) como un cuerpo de roca caracterizado por una particular combinación de litologías, estructuras físicas y biológicas que le confieren un aspecto diferente a los cuerpos de rocas adyacentes dentro de una secuencia.

Este artículo se enfoca en la descripción de rocas sedimentarias clásticas: psefíticas, psamíticas y pelíticas, presentando criterios para la distinción petrológica, especificación de las características de textura e identificación de estructuras sedimentarias, incluyendo en la descripción conceptos de asociación de facies y ambientes sedimentarios análogos descritos en James y Dalrymple (2010). En la documentación de litofacies considerada por Miall (2010), quien presenta las litofacies posibles en sistemas aluviales. Para la identificación de superficies de erosión se utilizó la discriminación presentada por Tedesco (2010).

La medición de paleocorrientes se la realizó a partir de la documentación de superficies de erosión y estructuras sedimentarias de transporte como son imbricación y sets con estratificación cruzada. Siguiendo a Tucker (2009), estos datos fueron analizados mediante una media geométrica estadística para definir la dirección de la paleocorriente. Medidas de GPS-diferencial se utilizaron para medir diferencias de alturas y estimar espesores.

En el levantamiento de datos en campo, se utilizó el GPs Trimble GeoXT 2008-Series, siguiendo los protocolos de recolección de datos reportados por Fontán et al., 2007; y para el procesamiento de datos se utilizó software ArcPad 8. Datos de alturas obtenidos del GPS-diferencial han sido integrados en la elaboración de columnas estratigráficas.

\section{RESULTADOS}

$\mathrm{Al}$ w del área de estudio areniscas y cuarzo-areniscas de la Formación Hollín afloran extensamente. En este sector, una de las principales características morfológicas es la presencia de deslizamientos rotacionales y gravitacionales; estos deslizamientos proporcionan detrito a la cuenca del río Yunganza. Mientras que en el flanco oriental del río, se observan andesitas y brechas volcánicas de la formación Misahuallí, en este sector no se documentan movimientos en masa (ver Figura 2).

La tabla 2 indica el resumen de facies documentadas en esta investigación. En las márgenes del río Yunganza, se documentó depósitos de terrazas aluviales (TA-1 y TA-2) y depósitos aluviales (DA), estos depósitos son escritos a continuación (ver Tabla 2).

\section{TERRAZA ALUVIALES 1 (TA-1)}

Afloran en los flancos altos del rio Yanganatza y se depositan cubriendo en discordancia a la Formación Hollín (ver Figura 2). El espesor máximo observado en campo alcanza los $62 \mathrm{~m}$ al w de la zona de estudio mientras que en el flanco sE del río Yunganza se observaron espesores máximos de $20 \mathrm{~m}$ (ver Figuras 3 y 4 ).

En la base de la secuencia, se observan apilaciones verticales, métricas, de tendencia estrato decreciente y grano decreciente, de estratos de morfología canalizada, lateralmente discontinua de conglomerados (LT-1: Fig. 4) oligomícticos, con clastos centimétricos, de 3 a $24 \mathrm{~cm}$, de areniscas, lutitas, andesitas, polimodales, 
Tabla 1. Coordenadas de afloramientos estudiados a detalle. Sistema de proyección WGS-84, 17S.

\begin{tabular}{lll}
\hline Afloramiento & UTM-X & UTM-Y \\
\hline A & $789517 \mathrm{E}$ & $9678321 \mathrm{~N}$ \\
B & $789850 \mathrm{E}$ & $9678236 \mathrm{~N}$ \\
C & $790268 \mathrm{E}$ & $9678215 \mathrm{~N}$ \\
D & $790453 \mathrm{E}$ & $9678580 \mathrm{~N}$ \\
E & $790485 \mathrm{E}$ & $9677744 \mathrm{~N}$ \\
F & $789676 \mathrm{E}$ & $9677786 \mathrm{~N}$ \\
G & $789480 \mathrm{E}$ & $9677490 \mathrm{~N}$ \\
\hline
\end{tabular}

Tabla 2. Resumen de litofacies

\begin{tabular}{|c|c|c|c|}
\hline Facies & Litología & Estructuras sedimentarias & Interpretación \\
\hline $\begin{array}{l}\text { LT-1 } \\
\text { Gmg, Gh, } \\
\text { Gt }\end{array}$ & $\begin{array}{l}\text { Conglomerados, oligomícticos, } \\
\text { con clastos de areniscas, lutitas, } \\
\text { andesitas, polimodales, matriz } \\
\text { soportado, de matriz arenosa. }\end{array}$ & $\begin{array}{l}\text { Gradación normal } \\
\text { Estratificación cruzada trans- } \\
\text { versal, } \\
\text { Imbricación }\end{array}$ & Flujos de escombros plásticos \\
\hline $\begin{array}{l}\text { LT-2 } \\
\text { Gmm,Gp }\end{array}$ & $\begin{array}{l}\text { Conglomerados, polimígnicos, } \\
\text { con clastos de areniscas, andesi- } \\
\text { tas, dacitas, riolitas, cuarcitas, fili- } \\
\text { tas, polimodales, clasto soportado, } \\
\text { de matriz arenosa. }\end{array}$ & $\begin{array}{l}\text { Masiva a ligeramente gradado, } \\
\text { ocasionalmente estratificación } \\
\text { cruzada planar }\end{array}$ & $\begin{array}{l}\text { Flujos de escombros pseudo- } \\
\text { plásticos }\end{array}$ \\
\hline $\begin{array}{l}\text { LT-3 } \\
\text { Gci, Gcm, } \\
\text { Gmm }\end{array}$ & $\begin{array}{l}\text { Conglomerados, oligomícticos, } \\
\text { ccon clastos de areniscas, y lutitas, } \\
\text { polimodales, clasto soportado. }\end{array}$ & Gradación inversa & \\
\hline $\begin{array}{l}\mathrm{LT}-4 \\
\text { St, Sr }\end{array}$ & $\begin{array}{l}\text { Arenisca gruesa a fina, con gránu- } \\
\text { los volcánicos, mal sorteada }\end{array}$ & $\begin{array}{l}\text { Estratificación cruzada planar, } \\
\text { ripples de corriente }\end{array}$ & Barras fluviales \\
\hline $\begin{array}{l}\mathrm{LT}-5 \\
\mathrm{Sl}, \mathrm{Sm}\end{array}$ & $\begin{array}{l}\text { Arenisca media a gruesa, con } \\
\text { clastos metamórficos y volcánicos, } \\
\text { moderadamente bien sorteada }\end{array}$ & $\begin{array}{l}\text { Masiva, ocasionalmente lamina- } \\
\text { ción cruzada }\end{array}$ & \\
\hline $\begin{array}{l}\text { LT-6 } \\
\text { Sp, St }\end{array}$ & $\begin{array}{l}\text { Arenisca muy gruesa a media, con } \\
\text { gránulos de cuarzo }\end{array}$ & $\begin{array}{l}\text { Estratificación cruzada trans- } \\
\text { versal y planar. } \\
\text { Gradación normal }\end{array}$ & \\
\hline $\begin{array}{l}\text { LT-7 } \\
\text { Fl, Sm, C }\end{array}$ & $\begin{array}{l}\text { Limolita arenosa, arenisca fina a } \\
\text { muy fina, con esporádicos gránu- } \\
\text { los volcánicos y carbón }\end{array}$ & $\begin{array}{l}\text { Masiva, ocasionalmente Small } \\
\text { ripples }\end{array}$ & Planicies de inundación \\
\hline
\end{tabular}

subangulosos, de esfericidad media, matriz soportado, de matriz areno-limosa, con gradación normal (Gmg: Fig. 5 y Fig. 6), estratificación cruzada transversal (Gt) e imbricación (Gci) con dirección NE-E (62: Fig. 3).

Hacia la parte media, se observan intercalaciones centimétricas, de: conglomerados (LT-2; Fig. 4), polimígnicos, con clastos subredondeados de esfericidad media, de areniscas, andesitas, dacitas, riolitas, cuarcitas, filitas, polimodales, clasto soportado, de matriz arenosa, masivos a ligeramente gradados (Gmm: Fig.
5 y Fig. 6), y estratificación cruzada planar (Gp: Fig. 5 y Fig. 6), con areniscas (LT-3: Fig. 4) de grano medio a grueso, con gránulos, subangulosos de esfericidad media, de cuarzo y fragmentos andesíticos, con estratificación cruzada transversal (St: Fig. 5 y Fig. 6) y estratificación cruzada planar (Sp; Fig. 5 y Fig. 6).

El tope de la secuencia es caracterizado por la presencia de niveles de limolitas arenosas (LT-3: Fig. 4), masivas (FL: Fig. 5 y Fig. 6) con fragmentos de carbón (C), distribuidos de manera periclinal entre estratos 
centimétricos de microconglomerados de cuarzo, matriz soportada.

$\mathrm{Al}$ analizar la composición de los clastos presentes en TA-1, se observa que existe un dominio de clastos de cuarzo (50\%), areniscas limosas (35\%), lutitas (10\%) $\mathrm{y}$ andesitas (5\%).

Interpretación. Miall (1988) asocia a facies conglomeráticas gradadas a flujos de escombros. El grado de sorteo y la morfología de los clastos documentados indican que la fuente de detrito es distal. A partir de la medición de paleocorriente se observa que la fuente de detrito se ubica en el flanco $\mathrm{W}$ del río. Por otro lado, Brierley, Liu y Crook (1993) indican que niveles de areniscas gruesas con estratificación cruzada, distribuidas en facies de conglomerados se forman debido al imput periódico de detrito en abanicos aluviales desde bloques cercanos; por lo cual estas facies son interpretadas como flujos de escombros. distales provenientes de bloques del $\mathrm{w}$, asociados a la Formación Hollín, en función de la composición de clastos documentados.

\section{TERRAZA ALUViALES 2 (TA-2)}

Cubren en discordancia a los depósitos TA-1 (ver Figura 2). El espesor máximo observado se aproxima a $80 \mathrm{~m}$ (ver Figuras 3 y 4). En la base de la secuencia se observan intercalaciones de paquetes métricos, canalizados, de conglomerados (LT-1; Fig. 4), oligomígticos con clastos redondeados de baja esfericidad, de cuarzo $\mathrm{y}$ andesitas, matriz soportado, de matriz arenosa, con gradación normal (Gmg: Fig. 5 y Fig. 6) y masivos $(\mathrm{Gmm})$, entre abundantes niveles con estratificación cruzada transversal (Gt: Fig. 6). Paleocorrientes indican una dirección de transporte hacia el N (ver Figura 3).

La parte media de esta secuencia es caracterizada por la presencia de areniscas (LT-5: Fig. 4) de grano grueso a muy grueso con clastos, subredondeados de baja esfericidad, andesíticos, mal sorteados, con gránulos subredondeados de esfericidad media, con estratificación cruzada paralela (Sp: Fig. 5 y Fig. 6) y estratificación cruzada transversal (St: Fig. 5 y Fig. 6); es común observar la presencia de estratos planares, de arenisca finas a muy finas, masivas $(\mathrm{Sm})$ con gránulos de cuarzo.

Hacia el tope de la secuencia, se observan esporádicos niveles conglomeráticos con abundantes fragmentos de areniscas y gránulos de cuarzo, imbricados en dirección NE-E. Niveles de areniscas (LT-6: Fig. 4) finas masivas (Sm: Fig. 5 y Fig. 6) con fragmentos de carbón (C) y limolitas arenosas con abundantes ripples (Sr).

El análisis modal de componentes clásticos en TA2 , indican un dominio de cuarzo (80\%), fragmentos volcánicos, andesitas, dacitas y riolitas (10\%). Se ob- servan también bloques conglomeráticos asociados a TA-1 (7\%) y en menor proporción fragmentos metamórficos (3\%).

Interpretación. Delcaillau et al., (2010) estudian depósitos estratificados de pie de talud en el alto de Marrakech y describe facies arenosas con estratificación cruzada entre facies de conglomerados masivos y gradación normal, con un clásico mal sorteo y patrones de baja esfericidad, esta descripción concuerda con características litológicas y morfológicas observadas en TA-2.

La dirección de paleocorrientes documentada en TA-2 cambia de N-NE a N con respecto a la dirección documentada en los depósitos de TA-1 (ver Figuras 3 y 4). Fontana, Mozzi y Marchetti, (2014) indican que en facies de conglomerados de baja esfericidad y mal sorteo son indicativos de retrabajamiento de flujos de escombros precedentes.

\section{DEPósitos ALUViales (DA)}

Cubren en discordancia a los TA-1 y TA-2. Buenas exposiciones de esta secuencia se encuentran a orillas del río Yungantza. Corresponden a barras fluviales y planicies de inundación activas; las barras fluviales están formadas por conglomerados matriz soportados, polimígticos, estratificados, con gradación normal, hacia el tope se observan niveles de areniscas gruesas a medias, bien sorteadas, con estratificación cruzada transversal y paralela. Planicies de inundación están compuestas por horizontes de areniscas finas a medias, con niveles de limolitas arenosas con abundante materia orgánica. En la actualidad, la dirección de transporte del río indica una tendencia $\mathrm{N}$.

La composición modal de DA es dominada por fragmentos volcánicos de composición andesítica (50\%), dacítica (20\%), fragmentos metamórficos de cuarcitas (10\%), filitas (7\%) y fragmentos sedimentarios de cuarzo (5\%), caliza (5\%) y lutitas (3\%).

\section{DISCUSIón}

A escala regional, abanicos aluviales se desarrollan en los flancos de las cordilleras y se forman a partir del depósito de detrito que se generan por erosión de zonas altas de los cinturones montañosos (Platt y Keller, 1992). La forma cónica de los abanicos aluviales es el resultado de la interacción entre el cambio de pendiente y la pérdida de energía del flujo que arrastra sedimento (Brierley et al., 1993).

La cantidad de detrito disponible para la sedimentación en abanicos aluviales depende directamente de los procesos erosivos y los patrones de sedimentación en las microcuencas alimentadoras del sistema fluvial que forma el abanico aluvial (Reitz y Jerolmack, 2012). 


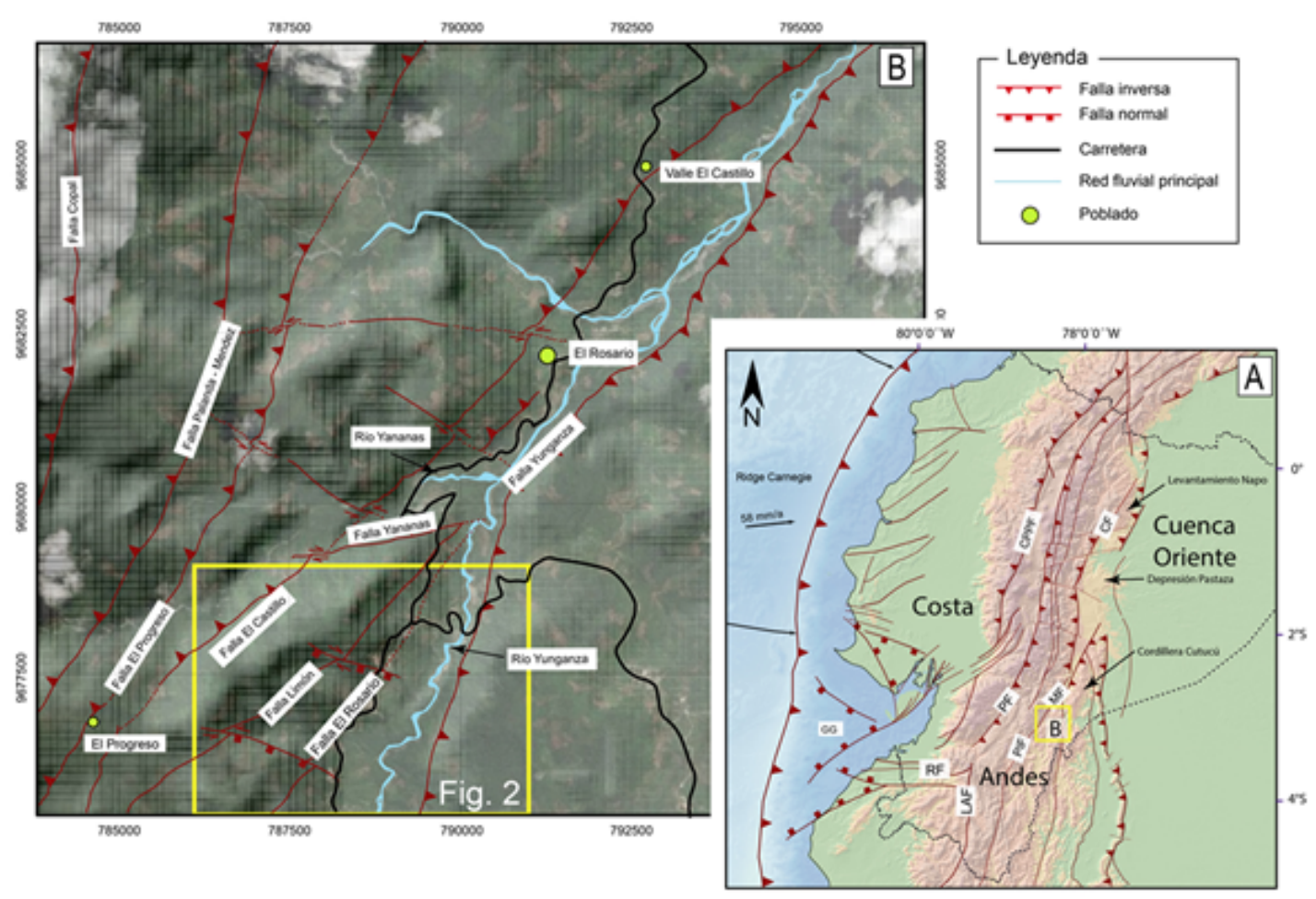

Figura 1. Esquema tectónico regional

A. Marco tectónico del Ecuador; B. Esquema estructural regional de la zona de estudio, modificado de IIGE, (2019).

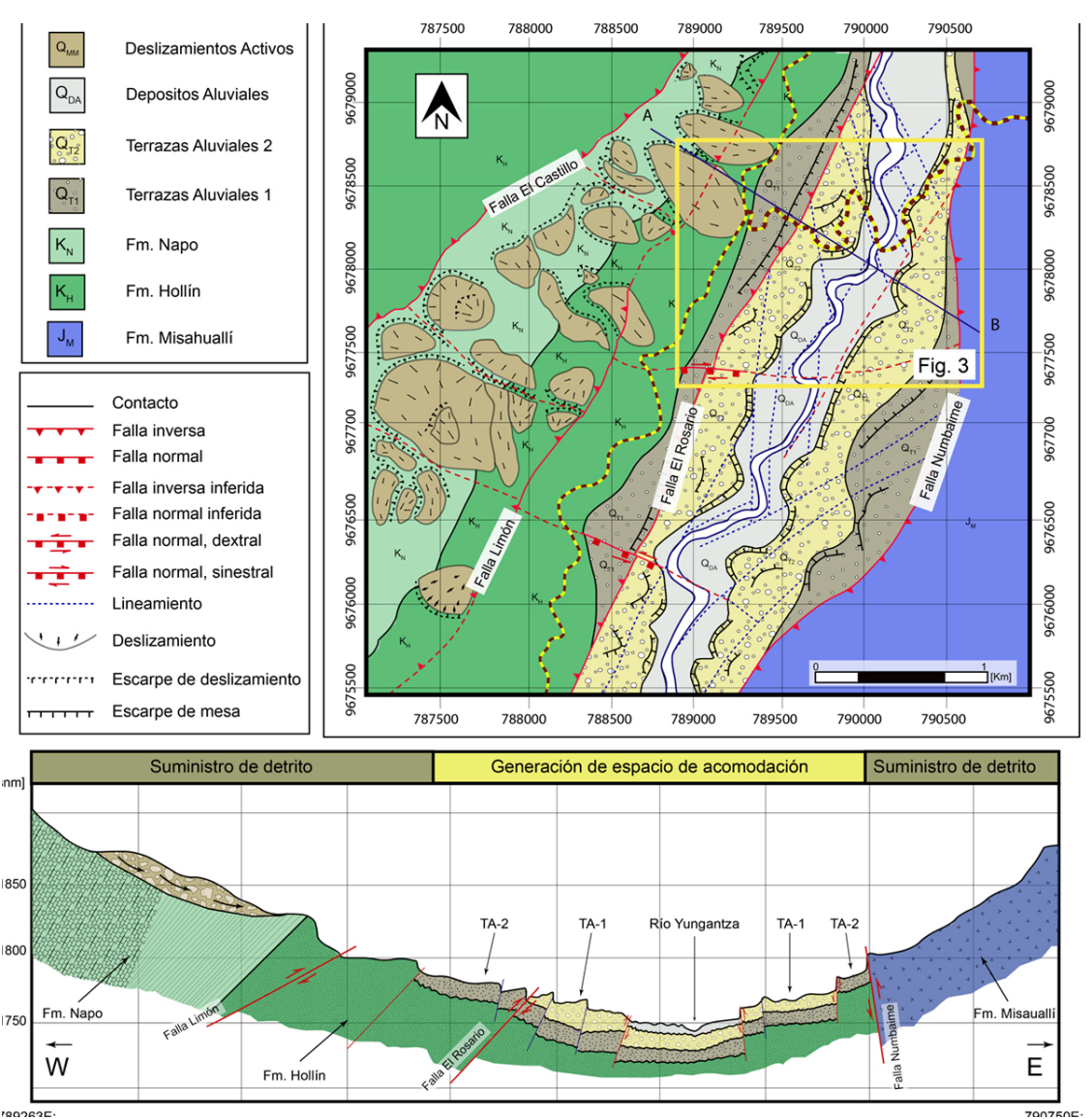

Figura 2. Mapa geológico de la zona de estudio

Fuente: Modificado de IIGE, (2019). 


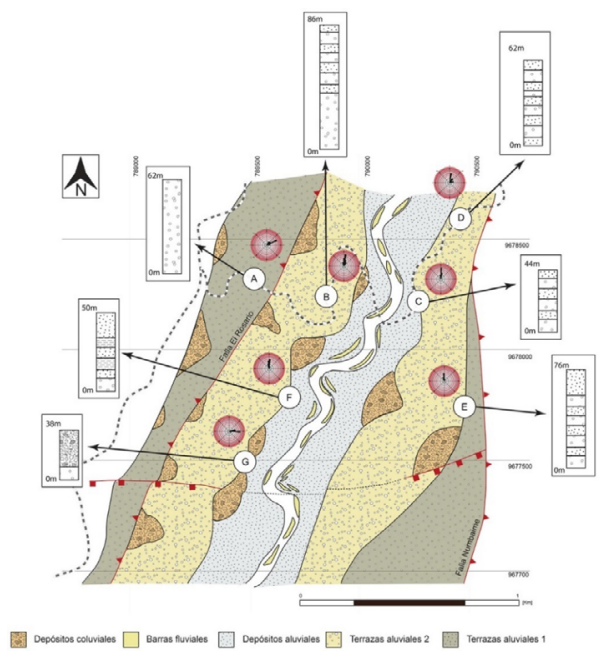

Figura 3. Mapa de terrazas aluviales y depósitos aluviales del río Yunganza al sur del sector Rosario.

En la figura, se observan los afloramientos estudiados a detalle y señalados en la tabla 1 , dirección media de paleocorrientes y espesor estimado de cada secuencia.
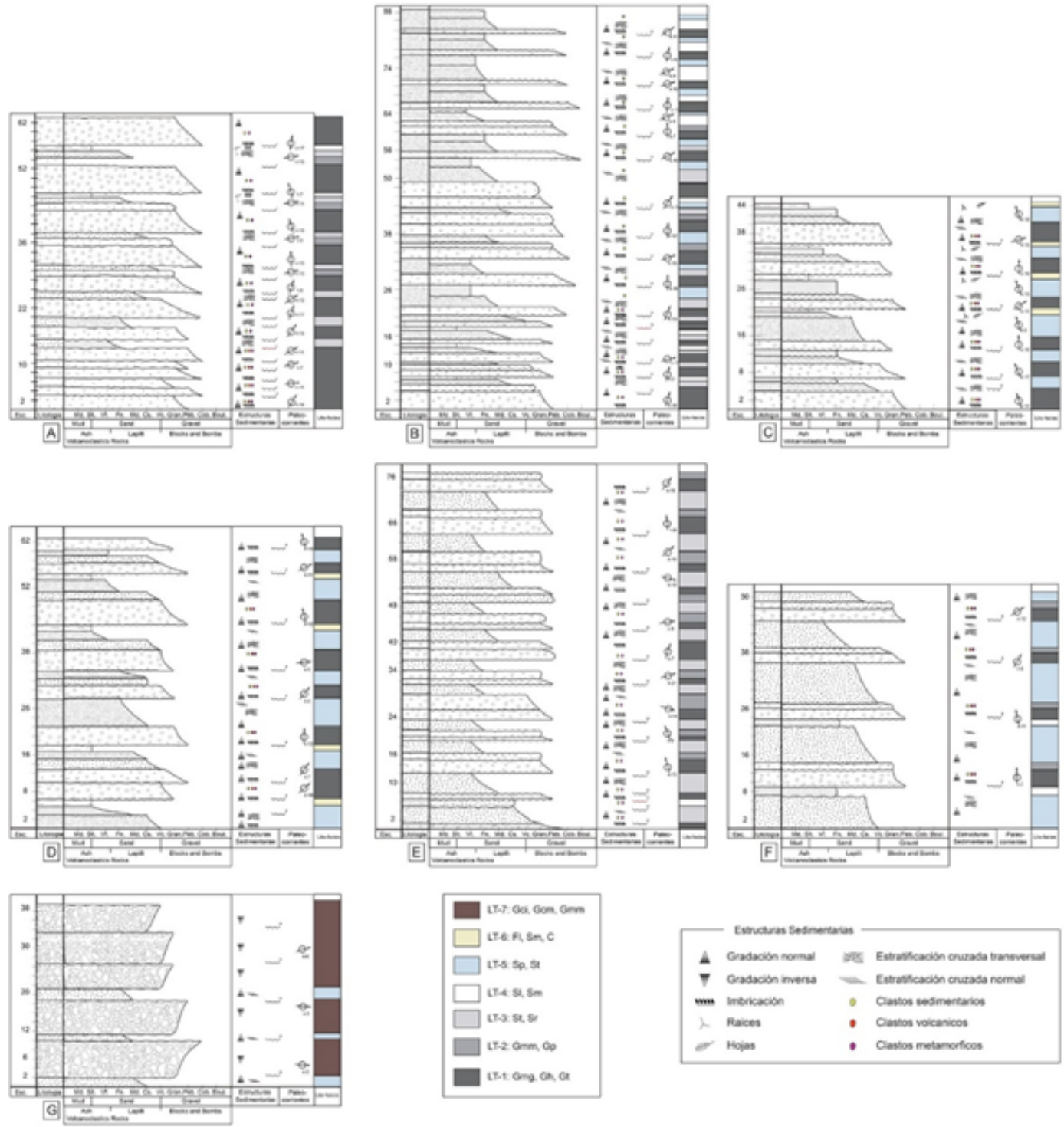

Figura 4. Columnas estratigráficas de terrazas aluviales.

En la figura, se observan las columnas estratigráficas de los afloramientos estudiados a detalle señalados en la tabla 1 y en la figura 3. 
Figura 5. Afloramientos tipo de terrazas en el río Yunganza.

A. Canales conglomeráticos con imbricación, estratificación cruzada transversal (Gt) y gradación normal, entre niveles de areniscas medias a gruesas con estratificación cruzada paralela (Sp) de T-A-1 (A-UTM X: 789 517E; Y: $9678321 \mathrm{~N}$ ); B. Barras de arenisca media a fina con estratificación cruzada transversal (ST), entre canales conglomeráticos, TA-1 (B-UTM X: 789 850E; Y: 9678 $236 \mathrm{~N})$; C. Macroformas de barras de arena (SB) y barras de graba en TA-2 (E-UTM X: 790 485E; Y: $9677744 \mathrm{~N}$ ); D. Litofacies de areniscas gruesas con estratificación cruzada transversal, entre conglomerados masivos y gradación normal TA-2 (D-UTM X: 790 453E; Y: 9 678580N).
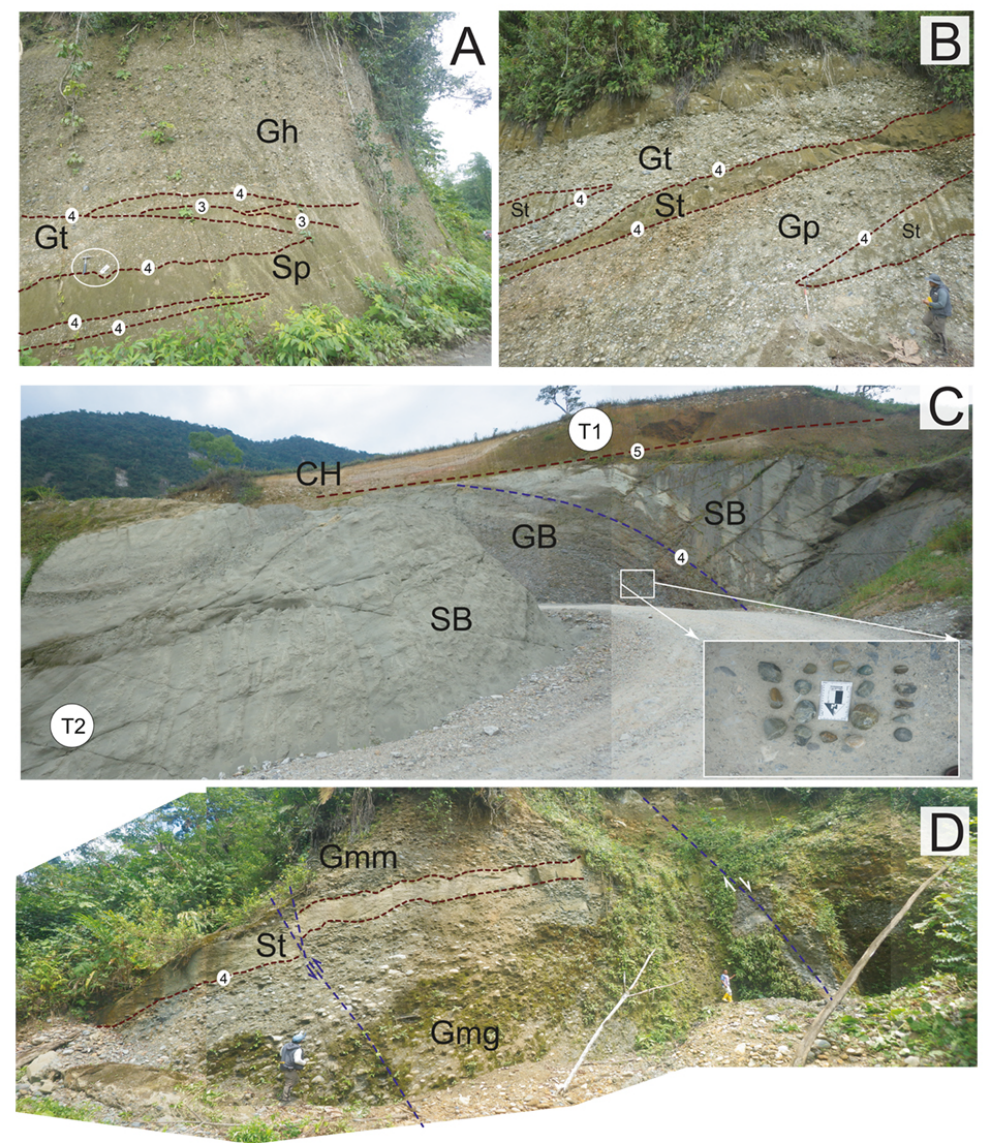
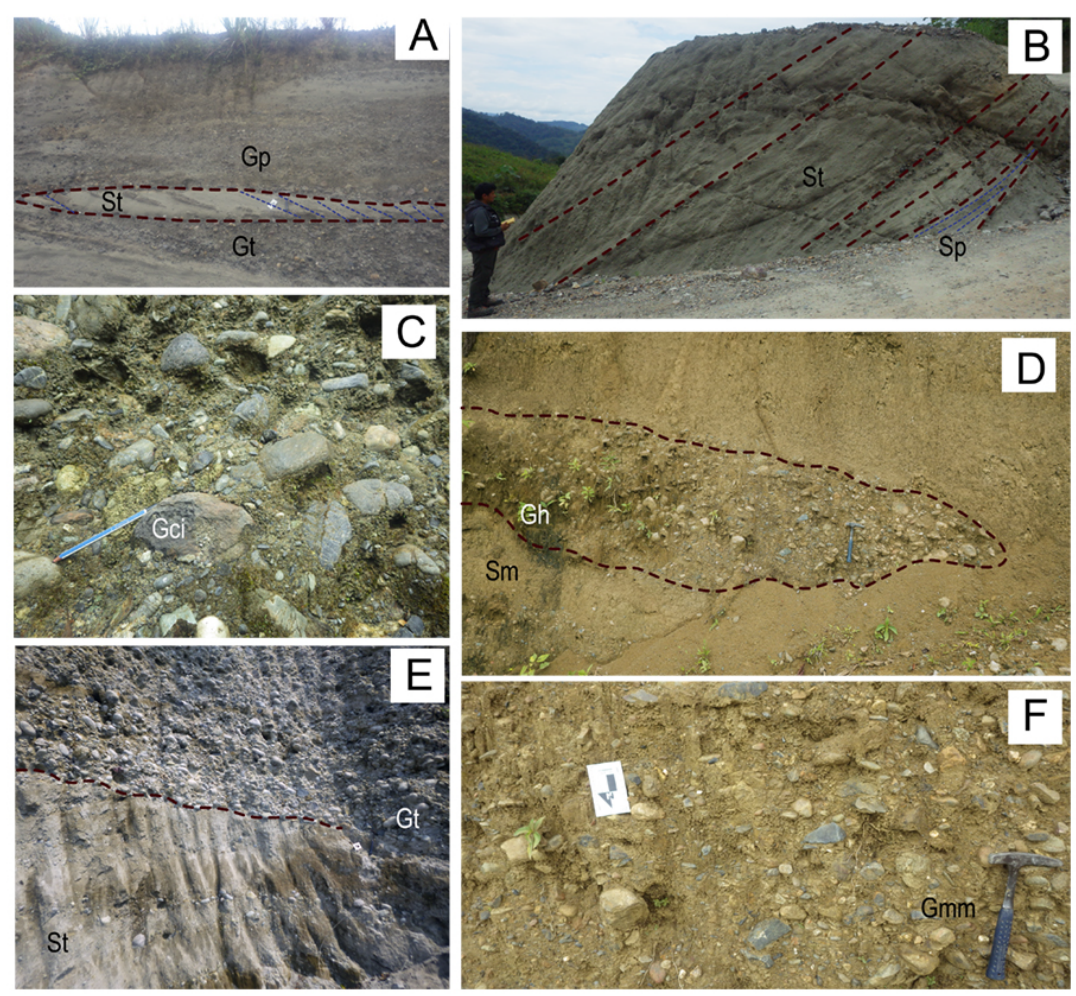

Figura 6. Litofacies tipo de terrazas aluviales del río Yunganza.

A. Barras de arena (St) entre canales conglomeráticos (Gp-Gt), TA-2. B. Planicies de arena (St-Sp), TA-2. C. Facies conglomeráticas (Gci), TA-1. D. Canales conglomeráticos aislados $(\mathrm{Gh})$ entre planicies de arena $(\mathrm{Sm})$, TA-1. E. Facies conglomeráticas imbricadas (Gt) cubriendo a barras de arena (St), TA-1; F. Flujos de escombros masivos (Gmm), DA. 


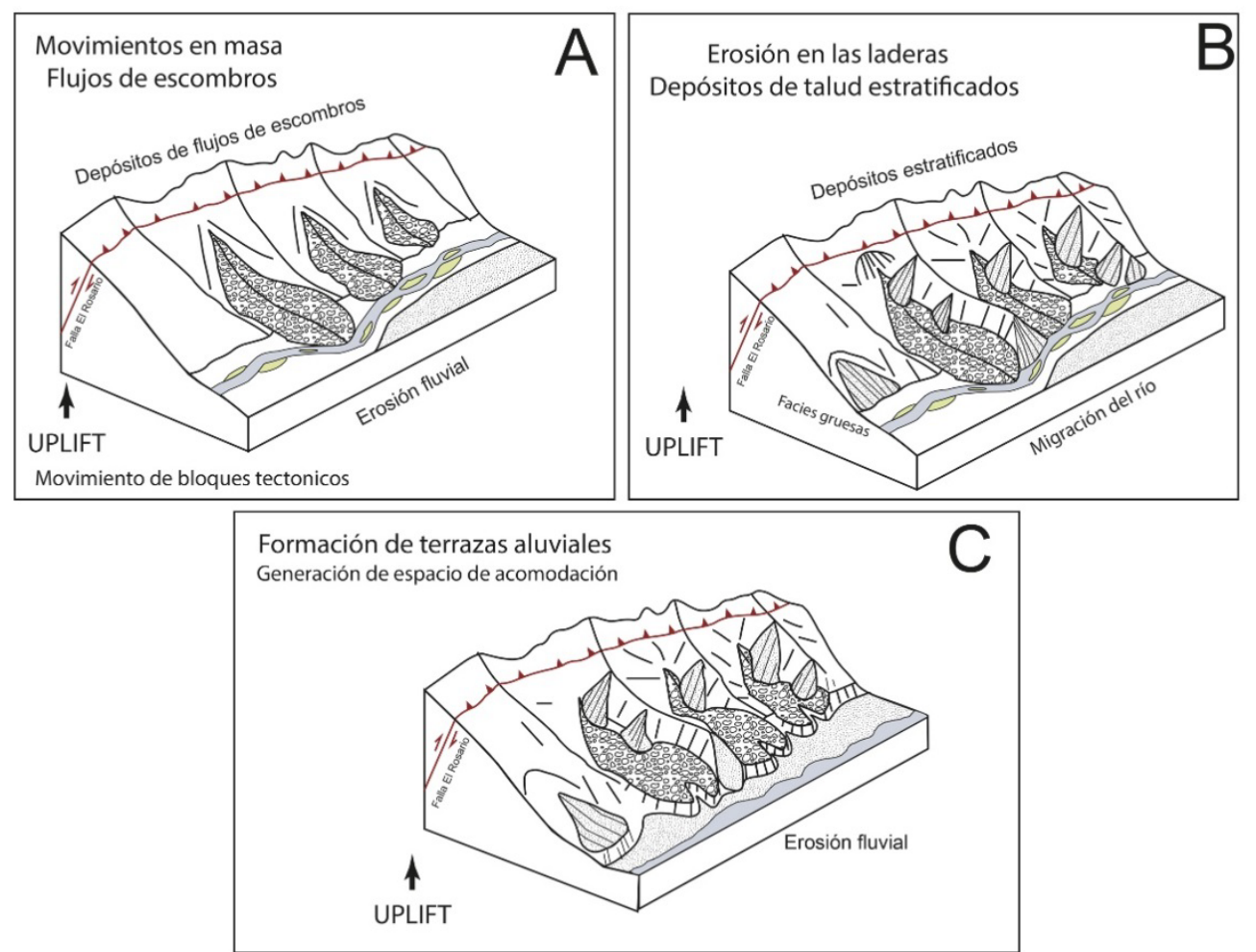

Figura 7. Modelo de generación de detrito y formación de terrazas aluviales en el río Yunganza. Elaborado a partir de la interpretación de ambientes de depósito, considerado a Decaillau, et al. (2010) y Ventra y Clarke (2018).

Los procesos de erosión están vinculados a la denudación física por factores climáticos de la roca fuente; sin embargo, la erosión implica patrones de sedimentación iniciales que permiten que el sedimento pueda ser transportado por los sistemas fluviales de una determinada región a otra (Latrubesse, 2015).

En la región subandina ecuatoriana, los patrones de sedimentación de sistemas fluviales no han sido descritos en trabajos anteriores. El rio Yunganza es un río subandino, de dirección NE, alimentador del río Santiago, que transporta grandes cantidades de detrito desde la región subandina a la planicie amazónica.

En el flanco o del río Yunganza, movimientos de bloques tectónicos deforman principalmente a rocas de la Formación Hollín, factores climáticos ocasionan la degradación de estas rocas y generan movimientos en masa, cuando un movimiento en masa es activado se generan flujos de escombros (TA-1) que rellenan los flancos occidentales del río (ver Figura 7A).

Depósitos estratificados de pie de talud (TA-2) se forman por erosión de flujos de escombros y laderas próximas (ver Figura 7B). La documentación de paleocorrientes en TA-1 y TA-2 permite indicar la migración del río desde el flanco o hacia su posición actual. La erosión generada por el río a través de su historia reciente se produce en TA-1 у TA-2; el grado de incisión en TA- 1 corresponde a $54 \mathrm{~m}$, mientras que en la TA-2 se midieron tasas de erosión de $10 \mathrm{~m}$; lo que indica un intenso reciclaje de detrito en TA-1.

La migración del canal activo del río Yunganza erosiona flujos de escombros y genera de detrito disponible para la sedimentación. En las márgenes del río, el suministro de detrito es caracterizado por deslizamientos gravitacionales, mientras que en los flancos altos del río el suministro de sedimento es caracterizado por deslizamientos compuestos. De esta manera, los patrones de sedimentación en el rio Yunganza implica la formación de terrazas aluviales a partir del retrabajamiento de flujos de escombros y depósitos aluviales (ver Figura 7C).

Según Sass y Krautblatter (2007), depósitos estratificados de talud indican la interacción entre factores climáticos y material no consolidado, sobre una pendiente de alto ángulo. Delcaillau et al., (2010) indican que una característica genética de estos depósitos es la intercalación de periodos de exhumación de bloques entre periodos de relativa tranquilidad tectónica, los cuales permiten el transporte de flujos sobresaturados en sedimento. En el flanco occidental del río Yunganza, depósitos estratificados de talud, alcanzan espesores de $20 \mathrm{~m}$ en TA- 2 .

\section{CONCLUSIONES}

- Se identificaron 14 litofacies: Gcm, Gci, Gmg, Gmm, 
Gt, Gp, Gh, Sp, St, Sr, Sl, Sm, Fl, C agrupadas en 7 macroformas descritas en 7 facies.

- Las facies LF-1 y LF-2 son utilizadas para describir facies de flujos de escombros distales, que presentan grados variables de sorteo y permitieron el desarrollo de estructuras sedimentarias de transporte de detrito como son: imbricación, gradación y estratificación cruzada; mientras que la litofacies LF-3 es utilizada para describir flujos de escombros proximales, característicamente masivos, no sorteados.

- Las facies LF-4, LF-5 y LF-6 son utilizadas para describir macroformas de origen fluvial como son canales y barras - longuitudinales y transversales- que caracterizan a depósitos de canal activo en sistemas fluviales confinados. Mientras que la facie LF-7 es utilizada para describir macroformas de planicies de inundación.

- En el río Yunganza, los procesos de sedimentación responden a la generación de flujos de escombros desde el flanco occidental del río en donde aflora extensamente la Formación Hollín. En lo posterior, estos flujos de escombros son erosionados tanto por acción de la gravedad como por erosión fluvial. De esta manera, la formación de terrazas aluviales responde a movimientos de bloques tectónicos a lo largo del flanco oriental de la cordillera Real.

- Los procesos de erosión en el río Yunganza están relacionados al transporte de sedimento, esto incluye: la generación de flujo de escombros, depósitos estratificados de talud, en lo posterior la erosión de flujos de escombros y construcción de terrazas aluviales que son a su vez, retrabajadas en periodos de migración del rio relacionados a tasas de exhumación del bloque occidental del rio Yunganza.

\section{REFERENCIAS}

Aspden, J. A. and Litherland, M. 1992. The geology and Mesozoic collisional history of the Cordillera Real, Ecuador. Tectonophysics, 205(1-3), 187-204. Doi. org/10.1016/0040-1951(92)90426-7

Baby, P., Rivadeneira, M., Barragán, R. and Christophoul, F. 2013. Thick-skinned tectonics in the Oriente foreland basin of Ecuador. Geological Society, London, Special Publications, 377(1), 59-76. Doi.org/10.1144/SP377.1

Benito, G., Gutiérrez, F., Pérez-González, A. and Machado, M. J. 2000. Geomorphological and sedimentological features in Quaternary fluvial systems affected by solution-induced subsidence (Ebro Basin, NE-Spain). Geomorphology, 33(34), 209-224. Doi.org/10.1016/S0169-555X(99)00124-5

Brierley, G. J., Liu, K. and Crook, K. A. 1993. Sedimentology of coarse-grained alluvial fans in the Markham Valley, Papua New Guinea. Sedimentary Geology, 86(3-4), 297 324. Doi.org/10.1016/0037-0738(93)90027-3
Bull, W.B., 1977. The alluvial fan environments. Prog. Phys. Geogr. 1, 222e270

Delcaillau, B., Laville, E., Amhrar, M., Namous, M., Dugué, O. and Pedoja, K. 2010. Quaternary evolution of the Marrakech High Atlas and morphotectonic evidence of activity along the Tizi N'Test Fault, Morocco. Geomorphology, 118(3-4), 262-279. Doi.org/10.1016/j. geomorph.2010.01.006

Fontán, A., Alcántara-Carrió, J., Poveda, J. M., \& Peña, M. A. 2007. Aplicación de técnicas de GPS diferencial, fotogrametría y geofísica a la cuantificación de procesos erosivos y balances sedimentarios en playas y dunas costeras. Teledetección-Herramienta para la gestión sostenible. Proc XII Congr Asociación Española de Teledetección, 19-21. ISBN: 975-987-543-127-0

Fontana, A., Mozzi, P. and Marchetti, M. 2014. Alluvial fans and megafans along the southern side of the Alps. Sedimentary Geology, 301, 150-171.

Doi.org/10.1016/j.sedgeo.2013.09.003

IIGE, 2019. Memoria Técnica de la HG-Mendez, esc. 1:100.000. Informe no publicado.

James, N.P. and Dalrymple R., W. 2010. Facies Models 4. GEotext 6. Geological Association of Canada.

Jolley, E. J., Turner, P., Williams, G. D., Hartley, A. J. and Flint, S. 1990. Sedimentological response of an alluvial system to Neogene thrust tectonics, Atacama Desert, northern Chile. Journal of the Geological Society, 147(5), 769-784. Doi.org/10.1144/gsigs.147.5.0769

Latrubesse, E. M. 2015. Large rivers, megafans and other Quaternary avulsive fluvial systems: A potential "who's who" in the geological record. Earth-Science Reviews, 146, 1-30.

Lewin, J., Macklin, M. G. and Johnstone, E. 2005. Interpreting alluvial archives: sedimentological factors in the British Holocene fluvial record. Quaternary Science Reviews, 24(16-17), 1873-1889. Doi.org/10.1016/j. quascirev.2005.01.009

Litherland, M. 1994. The metamorphic belts of Ecuador. British Geological Survey, Overseas Memoir., 11.

Miall, A. D. 1988. Facies architecture in clastic sedimentary basins. In New perspectives in basin analysis (pp. 67-81). Springer, New York, NY. Doi.org/10.1007/978-1-46123788-4_4

Miall, A. D. Alluvial deposits 2010 Facies Models, 4. Geological Association of Canada, St. John's, Newfoundland, 105-137.

Platt, N. H. and Keller, B. 1992. Distal alluvial deposits in a foreland basin setting-the Lower Freshwater Miocene), Switzerland: sedimentology, architecture and palaeosols. Sedimentology, 39(4), 545-565. Doi.org/10.1111/j.1365-3091.1992.tb02136.x

Pratt, W. T., Duque, P. and Ponce, M. 2005. An autochthonous geological model for the eastern Andes of Ecua- 
dor. Tectonophysics, 399(1-4), 251-278. Doi.org/10.1016/j. tecto.2004.12.025

Reitz, M. D. and Jerolmack, D. J. 2012. Experimental alluvial fan evolution: Channel dynamics, slope controls, and shoreline growth. Journal of Geophysical Research: Earth Surface, 117(F2). Doi.org/10.1029/2011JF002261

Riel, N., Guillot, S., Jaillard, E., Martelat, J. E., Paquette, J. L., Schwartz, S., Goncalves P., Ducaux, N., Thebaud N., Lanari P., Janots E. and Yuquilema J. 2013. Metamorphic and geochronogical study of the Triassic El Oro metamorphic complex, Ecuador: Implications for high-temperature metamorphism in a forearc zone. Lithos, 156, 41-68. Doi.org/10.1016/j.lithos.2012.10.005

Sage, F., Collot, J. Y. and Ranero, C. R. 2006. Interplate patchiness and subduction-erosion mechanisms: Evidence from depth-migrated seismic images at the central Ecuador convergent margin. Geology, 34(12), 997-1000. Doi.org/10.1130/G22790A.1

Sass, O. and Krautblatter, M. 2007. Debris flow-dominated and rockfall-dominated talus slopes: Genetic models derived from GPR measurements. Geomorphology, 86(1-
2), 176-192. Doi.org/10.1016/j.geomorph.2006.08.012 Tedesco, A., Ciccioli, P., Suriano, J. and Limarino, C. O. 2010. Changes in the architecture of fluvial deposits in the Paganzo Basin (Upper Paleozoic of San Juan province): an example of sea level and climatic controls on the development of coastal fluvial environments. Geologica acta, 8(4), 0463-482. DOI: 10.1344/105.000001583 Tucker, M. E. (Ed.). 2009. Sedimentary petrology: an introduction to the origin of sedimentary rocks. John Wiley \& Sons. Ventra, D. and Clarke, L. E. 2018. Geology and geomorphology of alluvial and fluvial fans: current progress and research perspectives. Geological Society, London, Special Publications, 440(1), 1-21. Doi.org/10.1144/SP440.16

Walker, R. G. 1990. Facies modeling and sequence stratigraphy. Journal of Sedimentary Research, 60(5), 777-786.

Weissmann, G. S., Hartley, A. J., Nichols, G. J., Scuderi, L. A., Olson, M., Buehler, H. and Banteah, R. 2010. Fluvial form in modern continental sedimentary basins: distributive fluvial systems. Geology, 38(1), 39-42. Doi. org/10.1130/G30242.1 